\title{
Transcranial Direct Current Stimulation over Right Dorsolateral Prefrontal Cortex Enhances Error Awareness in Older Age
}

\author{
Siobhán Harty, ${ }^{1}$ Ian H. Robertson, ${ }^{1}$ Carlo Miniussi, ${ }^{2}$ Owen C. Sheehy, ${ }^{1}$ Ciara A. Devine, ${ }^{1}$ Sarahjane McCreery, ${ }^{1}$ \\ and Redmond G. $0^{\prime}$ Connell ${ }^{1}$ \\ ${ }^{1}$ Trinity College Institute of Neuroscience and School of Psychology, Trinity College Dublin, Dublin 2, Ireland, and ${ }^{2}$ Department of Clinical and \\ Experimental Sciences, University of Brescia, and Istituti di Ricovero e Cura a Carattere Scientifico Centro San Giovanni di Dio Fatebenefratelli, Brescia, \\ Italy
}

The ability to detect errors during cognitive performance is compromised in older age and in a range of clinical populations. This study was designed to assess the effects of transcranial direct current stimulation (tDCS) on error awareness in healthy older human adults. tDCS was applied over DLPFC while subjects performed a computerized test of error awareness. The influence of current polarity (anodal vs cathodal) and electrode location (left vs right hemisphere) was tested in a series of separate single-blind, Sham-controlled crossover trials, each including 24 healthy older adults (age 65-86 years). Anodal tDCS over right DLPFC was associated with a significant increase in the proportion of performance errors that were consciously detected, and this result was recapitulated in a separate replication experiment. No such improvements were observed when the homologous contralateral area was stimulated. The present study provides novel evidence for a causal role of right DLPFC regions in subserving error awareness and marks an important step toward developing tDCS as a tool for remediating the performance-monitoring deficits that afflict a broad range of populations.

Key words: cognitive aging; error awareness; performance monitoring; prefrontal cortex; tDCS

\section{Introduction}

The ability to monitor ongoing performance for occasional errors is essential for adaptive functioning in everyday life. Although young healthy adults are typically good at detecting their errors and adjusting their behavior accordingly, this capacity declines with age (Rabbitt, 1990; Harty et al., 2013) and is also compromised in many clinical conditions (O'Keeffe et al., 2007; Hester et al., 2009; O'Connell et al., 2009; David et al., 2012). The present study had the dual goals of establishing whether right DLPFC plays a causal role in supporting detection of performance errors ("error awareness") and evaluating the potential of transcranial direct current stimulation (tDCS) for remediating awareness deficits in older age.

Although there is a consensus across clinical, brain lesion, and neuroimaging studies that the capacity to monitor cognitive performance relies on a broadly distributed network of brain regions that includes prefrontal, parietal temporal, and insular cortices

Received Dec. 19, 2013; revised Jan. 14, 2014; accepted Jan. 29, 2014.

Author contributions: S.H., I.H.R., C.M., and R.G.O. designed research; S.H., O.C.S., C.A.D., S.M., and R.G.O. performed research; S.H. and R.G.O. analyzed data; S.H., I.H.R., and R.G.O. wrote the paper.

This work was supported by the Neuro-Enhancement for Independent Lives programme. We thank Christian Ruff for advice during the design of the study and Richard Carson for helpful comments during the preparation of the manuscript.

The authors declare no competing financial interests.

Correspondence should be addressed to Siobhán Harty, Trinity College Institute of Neuroscience, Lloyd Building, Trinity College Dublin, Dublin 2, Ireland. E-mail: sharty@tcd.ie.

DOI:10.1523/JNEUROSCI.5308-13.2014

Copyright $@ 2014$ the authors $\quad 0270-6474 / 14 / 343646-07 \$ 15.00 / 0$
(Klein et al., 2007; Hester et al., 2009), the literature is divided regarding the relative importance of right DLPFC. On the one hand, several studies have demonstrated a strong association between poor awareness of cognitive functioning and hypoperfusion and hypometabolism of right DLPFC in neurodegenerative diseases, such as Alzheimer's disease and frontotemporal dementia (Reed et al., 1993; Starkstein et al., 1995; Antoine et al., 2004; Harwood et al., 2005; Mendez and Shapira, 2005). Similarly, awareness of impairment in schizophrenia patients is correlated with right, but not left, DLPFC volume (Shad et al., 2004, 2006). These findings are also consonant with structural and functional data pointing to a specific role of right lateral prefrontal regions in mediating metacognitive abilities in young healthy adults (Fleming et al., 2010, 2012; De Martino et al., 2013). On the other hand, a role for right DLPFC was not supported by a number of eventrelated imaging studies investigating functional activations associated with error awareness (Debener et al., 2005; Hester et al., 2005, 2009; Klein et al., 2007).

The neural underpinnings of diminished error awareness in older age have not been established, but given that right DLPFC is one of the regions that undergoes the most volumetric changes with age (Hedden and Gabrieli, 2004; Raz, 2004), it is a plausible candidate structure. tDCS represents a potentially powerful tool, not only for investigating the contribution of brain regions to specific cognitive functions, but also for developing interventions to ameliorate cognitive deficits (Schulz et al., 2013). Here we report the results of a Sham-controlled crossover design experi- 
Table 1. Stimulation parameters and subject characteristics for each experiment

\begin{tabular}{|c|c|c|c|c|c|c|c|c|}
\hline \multirow[b]{2}{*}{ Experiment } & \multicolumn{2}{|c|}{ Stimulation } & \multicolumn{2}{|c|}{ Electrode location } & \multicolumn{4}{|c|}{ Subjects } \\
\hline & Name & Site & Anode & Cathode & $n$ & Age (years), mean (SD) & Mini-Mental State Examination, mean (SD) & Education (years) \\
\hline 1 & Anodal & Right DLPFC & F4 & $\mathrm{Cz}$ & 24 & $72.13(6.0)$ & $28.54(0.8)$ & $14.92(3.6)$ \\
\hline 3 & Cathodal & Right DLPFC & $\mathrm{Cz}$ & $\mathrm{F} 4$ & 24 & $69.71(4.2)$ & $28.54(1.4)$ & $14.58(3.5)$ \\
\hline 4 & Anodal & Right DLPFC & F4 & $\mathrm{Cz}$ & 24 & $72.08(5.7)$ & $28.75(0.9)$ & $14.17(3.6)$ \\
\hline
\end{tabular}

ment in which we tested the prediction that tonically stimulating right DLPFC of healthy older adults with anodal tDCS would lead to a significant improvement in error awareness as measured by the error awareness task (EAT) (Hester et al., 2005). We also conducted two follow-up experiments to explore whether there was evidence of hemispheric and current polarity specific effects. One further replication experiment was additionally performed to assess the reliability of the findings in the original experiment.

\section{Materials and Methods}

Subjects. In total, 106 healthy older adults 65-86 years of age were recruited for four separate experiments. All subjects were right-handed, had no metal implants, had normal or corrected-to-normal vision, and had no history of seizures, color blindness, or neurological illness. All subjects were asked to refrain from consuming caffeine on the day of each testing session. Four subjects were excluded because their Mini-Mental State Examination (Folstein, 1975) score $(<24)$ indicated possible cognitive impairment. Four subjects were excluded because their error awareness performance was at ceiling (100\%) for the Sham condition, and two were excluded for excessively poor error awareness $(>2.5 \mathrm{SD}$ below sample mean). One further subject was excluded for failing to abstain from caffeine consumption. As a result, the sample for Experiment 1 consisted of 24 subjects ( 14 female) with a mean age of 72.13 years (SD 6.0 years, range 65-86 years); the sample for Experiment 2 consisted of 24 subjects ( 13 female) with a mean age of 69.41 years (SD 4.3 years, range 65-80 years); the sample for Experiment 3 consisted of 24 subjects (16 female) with a mean age of 69.71 years (SD 4.2 years, range 65-84 years); and the sample for Experiment 4 consisted of 24 subjects ( 13 female) with a mean age of 72.08 years (SD 5.7 years, range $65-83$ years).

Procedures were approved by the ethical review board of the School of Psychology, Trinity College Dublin in accordance with the Declaration of Helsinki. Safety procedures based on noninvasive brain stimulation approaches were adopted (Poreisz et al., 2007; Rossi et al., 2009), and all subjects provided informed consent before the beginning of the experiment.

EAT. The EAT (Hester et al., 2005; Murphy et al., 2012) is a Go/ No-go response inhibition task in which subjects are presented with a serial stream of single-color words, with congruency between the semantic meaning of the word and its font color manipulated across trials. Subjects were trained to respond with a single-speeded left mouse button press in situations where the meaning of the word and the font color in which it was presented were congruent (Go trial) and to withhold this response when either of two different scenarios arose: (1) when the word presented on the current trial was the same as that presented on the preceding trial (Repeat No-go trial), and (2) when the meaning of the word and its font color did not match (Stroop No-go trial). In the event of a commission error (failure to withhold to either of these No-go trials), subjects were trained to signal their "awareness" by making a speeded right mouse button press. In these instances, they were not required to respond to the subsequent standard Go-trial, which was rendered irrelevant to guard against the possibility that some errors may fail to reach awareness because ongoing processing has been interrupted by the onset of another stimulus (Rabbitt, 2002).

Although previous work has demonstrated that performance accuracy and awareness rates are uncorrelated on the EAT (O'Connell et al., 2009), we used an adaptive staircase approach to maintain accuracy at a constant level to fully exclude the possibility that any observed changes in error awareness might be driven by an increase or decrease in the number of errors actually committed. In this approach, task difficulty is adaptively modified by varying the duration of stimulus presentation based on the subjects' accuracy over the preceding 40 trials. The first 40 stimuli of the task are always presented for $750 \mathrm{~ms}$ with an interstimulus interval (ISI) of $1250 \mathrm{~ms}$. The stimulus duration subsequently remains at $750 \mathrm{~ms}$ so long as accuracy on the previous 40 trials was between $50 \%$ and $60 \%$. If accuracy exceeds $60 \%$, the stimulus duration and ISI are set to $500 \mathrm{~ms}$ and $1500 \mathrm{~ms}$, respectively, for the subsequent 40 trials. If accuracy fell to $<50 \%$, the stimulus duration and ISI are both set to $1000 \mathrm{~ms}$. This evaluation and task adjustment occur every 40 trials thereafter. In a previous study, we have demonstrated that this adaptive staircase approach is successful in matching accuracy levels across young and older adults (Harty et al., 2013). All subjects performed five blocks of the task, consisting of 225 word presentations, 200 of which were Go trials and 25 of which were No-go trials (13 Repeat and 12 Stroop). Stimuli appeared $0.25^{\circ}$ over a white fixation cross and on a gray background. The duration of each block was $7.5 \mathrm{~min}$ after which the subject rested for $1 \mathrm{~min}$. Stimulus presentation was controlled by Presentation software (Neurobehavioural Systems). It was ensured that all subjects were well practiced and fully understood the requirements of the task before they began their first block of each testing session.

$t D C S$. Stimulation was delivered by a battery-driven DC Brain Stimulator Plus (NeuroConn), through a pair of $35 \mathrm{~cm}^{2}$ saline-soaked sponge electrodes. Current strength was $1 \mathrm{~mA}$ in all experiments. This produced current densities of $0.028 \mathrm{~mA} / \mathrm{cm}^{2}$ at the skin surface of the scalp.

In all four experiments, subjects underwent both Real and Sham tDCS in a single-blind, crossover manner. The order of the Real and Sham tDCS conditions was randomized, counterbalanced, and separated by a minimum of $6 \mathrm{~d}$ to reduce the risk of carryover effects. For the Real stimulation conditions, tDCS was applied for the duration of each of the five blocks of the EAT $(5 \times 7.5 \mathrm{~min})$ with a ramping period of $20 \mathrm{~s}$ at the onset and offset. No stimulation was applied during the rest periods between blocks. For the Sham conditions, the same electrode montage was used, but tDCS was only applied for $20 \mathrm{~s}$ at the beginning of each block, with a ramping period of $20 \mathrm{~s}$ at the onset and offset. This procedure ensured that, in both Real and Sham conditions, subjects experienced the same sensations associated with the onset of tDCS (e.g., tingling sensation) (Gandiga et al., 2006).

A summary of the stimulation parameters (guided by Ruff et al., 2013) and subject characteristics for each experiment are presented in Table 1. In Experiment 1, the anode served as the active electrode and was placed over the right DLPFC (F4, according to the 10-20 international system for electroencephalogram electrode placement). In Experiment 2, the anode was placed over the homologous contralateral area: left DLPFC (F3). In Experiment 3, the cathode served as the active electrode and was placed over the right DLPFC. Experiment 4 was a replication of Experiment 1 ; therefore, the anode again served as the active electrode and was placed over the right DLPFC. The reference electrode was placed over the vertex $(\mathrm{Cz})$ in all experiments.

At the end of both Real and Sham stimulation, we asked subjects to provide details on the sensations they experienced by completing a questionnaire developed by Fertonani et al. (2011).

Statistical analysis. An aware error was defined as any commission error after which subjects pressed the "awareness" button within 3000 ms. On a small minority of trials, subjects made a double press of the "go-trial" (left) button after a No-go trial. Given that it was not possible to determine whether double responses in these instances 
Table 2. Mean (SD) of performance indices on the EAT for real and sham stimulation for each experiment

\begin{tabular}{lll}
\hline & Real stimulation & Sham stimulation \\
\hline $\begin{array}{l}\text { Experiment 1 } \\
\text { Repeat accuracy (\%) }\end{array}$ & $75.96(12.18)$ & \\
Stroop accuracy (\%) & $68.12(13.80)$ & $75.56(15.47)$ \\
$\quad$ Repeat awareness (\%)* & $56.92(22.72)$ & $66.55(16.13)$ \\
$\quad$ Stroop awareness (\%) & $88.08(13.53)$ & $44.55(20.38)$ \\
Experiment 2 & & $89.14(11.39)$ \\
$\quad$ Repeat accuracy (\%) & $73.09(18.37)$ & \\
Stroop accuracy (\%) & $60.69(20.91)$ & $75.29(20.47)$ \\
Repeat awareness (\%) & $55.74(28.25)$ & $62.17(20.88)$ \\
Stroop awareness (\%) & $87.56(17.91)$ & $83.75(23.9)$ \\
Experiment 3 & & $87.06(14.67)$ \\
$\quad$ Repeat accuracy (\%) & $73.45(15.32)$ & $72.23(14.37)$ \\
Stroop accuracy (\%) & $60.57(16.57)$ & $61.49(16.99)$ \\
Repeat awareness (\%) & $64.81(16.94)$ & $68.07(21.96)$ \\
Stroop awareness (\%) & $93.97(7.41)$ & $92.03(9.13)$ \\
Experiment 4 & & \\
$\quad$ Repeat accuracy (\%) & $74.65(15.39)$ & $78.23(11.79)$ \\
Stroop accuracy (\%) & $64.52(17.08)$ & $65.95(14.35)$ \\
Repeat awareness $(\%)^{*}$ & $65.93(24.03)$ & $56.19(24.16)$ \\
Stroop awareness (\%) & $93.54(11.50)$ & $95.20(8.07)$ \\
\hline${ }^{*}<<0.05$. & &
\end{tabular}

reflected a failure to click the "awareness" (right) button in an attempt to signal an error, or an accidental double click of the "go-trial" button, we elected to exclude all such double responses from our calculation of error awareness. The effect of tDCS on error awareness and withholding accuracy were assessed using repeated-measures ANOVA. Given that previous studies using the EAT have identified better error awareness for Stroop compared with Repeat No-go trials (O'Connell et al., 2007, 2009; Hester et al., 2009), we analyzed the effect of tDCS on each of these trial types separately. We therefore included "Intervention" (Real versus Sham tDCS) and "No-go trial type" (Repeat vs Stroop) as within-subject factors. Significant interactions were followed up with paired samples $t$ tests. The effect sizes (Cohen's $d$ ) of Real compared with Sham tDCS were also computed. The effect of tDCS on all other EAT variables (i.e., mean Go-trial response time (ms); response time coefficient of variation (ms); mean stimulus duration (ms); mean awareness response time (ms)) was examined using one-way ANOVA. The data from Experiment 1 combined with the data from Experiment 4 (the replication experiment) afforded a sufficiently large sample to investigate whether variables, such as age (Ridding and Ziemann, 2010) and years of education (Berryhill and Jones, 2012), played a role in responsiveness to the tDCS intervention. Response to the intervention was indexed as the difference score between error awareness for Repeat No-go trials at Real versus Sham stimulation conditions. The Pearson's $r$ correlation coefficient between response to the intervention and age, and response to the intervention and years of education were calculated separately to assess the respective relationships. We were also interested in examining whether the effects of the intervention increased or decreased over the duration of the stimulation. To this end, we split performance on the EAT into two halves consisting of the first two blocks and the first half of the third block versus the last two blocks and the latter half of the third block. We then conducted an ANOVA with "Intervention" (Real vs Sham tDCS) and "Half" (First vs Second half) as within-subject factors.

\section{Results}

The means and SDs for performance indices on the EAT for Real and Sham stimulation in each of the experiments are provided in Table 2.

\section{Experiment 1: anodal stimulation over right DLPFC}

Real tDCS produced a significant improvement in error awareness compared with the Sham condition $\left(F_{(1,23)}=5.47, p=\right.$
$0.028, d=0.49)$. An Intervention $\times$ No-go trial type interaction $\left(F_{(1,23)}=4.31, p=0.049\right)$ was also observed, and paired sample $t$ tests indicated that the interaction was driven by a tDCS-induced improvement in error awareness for Repeat No-go trials $\left(t_{(23)}=\right.$ $2.49, p=0.020, d=0.51)$ that was absent for Stroop No-go trials $(p=0.709, d=-0.08$; Fig. 1$)$. There was no main effect of tDCS on withholding accuracy $(p=0.6)$, and there was no Intervention $\times$ No-go trial type interaction $(p=0.6)$, indicating that the adaptive adjustment of task difficulty was successful. There was also no effect of tDCS on mean Go-trial response time, response time coefficient of variation, or mean stimulus duration (all $p>$ 0.05). Therefore, anodal tDCS applied to the right DLPFC was associated with a specific improvement in error awareness for Repeat No-go trials.

Main effects of No-go trial type were observed for both error awareness $\left(F_{(1,23)}=177.29, p<.001\right)$ and withholding accuracy $\left(F_{(1,23)}=7.70, p=0.05\right)$. The former indicated significantly greater error awareness for Stroop compared with Repeat No-go trials across both Real and Sham sessions, whereas the latter indicated significantly greater withholding accuracy for Repeat compared Stroop No-go trials across both Real and Sham sessions. Both these main effects were recapitulated in the three subsequent experiments.

\section{Experiment 2: anodal stimulation over left DLPFC}

There was no main effect of intervention on error awareness ( $p=$ $0.7, d=-0.04)$, no Intervention $\times$ No-go trial type interaction $(p>0.05)$, and no significant effects on any other task variables (all $p>0.05)$.

\section{Experiment 3: cathodal stimulation over right DLPFC}

There was no main effect of intervention on error awareness $(p=$ $0.8, d=0.09)$, no Intervention $\times$ No-go trial type interaction $(p>0.05)$, and no significant effects on any other task variables (all $p>0.05)$.

\section{Experiment 4: anodal stimulation over right DLPFC (replication study)}

The results of Experiment 1 were recapitulated in Experiment 4 (Figure 2). tDCS produced an improvement in error awareness of comparable effect size with the main effect of Intervention $\left(F_{(1,23)}=3.61, p=0.07\right.$, two-tailed, $\left.d=0.33\right)$ and Intervention $\times$ No-go trial type interaction $\left(F_{(1,23)}=3.13\right.$, $p=0.09$, two-tailed) reaching trend levels of significance when two-tailed thresholds were applied, and reaching significance when one-tailed thresholds were applied. The paired sample $t$ tests indicated that tDCS produced a significant improvement in error awareness for Repeat No-go trials $\left(_{(23)}=\right.$ 2.05, $p=0.052, d=0.42$ ) that was absent for Stroop No-go trials $(p=0.5, d=-0.13)$. There was no effect of tDCS on any other task variables (all $p>0.05$ ). Therefore, anodal tDCS applied over the right DLPFC was once again associated with a specific improvement in error awareness for Repeat No-go trails.

When the data for Experiments 1 and 4 were combined, we observed a significant main effect of Intervention $\left(F_{(1,47)}=9.21, p=\right.$ $0.004, d=0.41)$ and a significant Intervention $\times$ No-go trial type interaction $\left(F_{(1,47)}=7.55, p=0.008\right)$ with follow-up tests indicating significant improvement in error awareness for Repeat No-go trials $\left(t_{(47)}=3.24, p=0.002, d=0.47\right)$ but no significant changes for Stroop No-go trials ( $p=0.5, d=-0.10$ ). Again, there was no effect of tDCS on any other task variables (all $p>0.05$ ). 


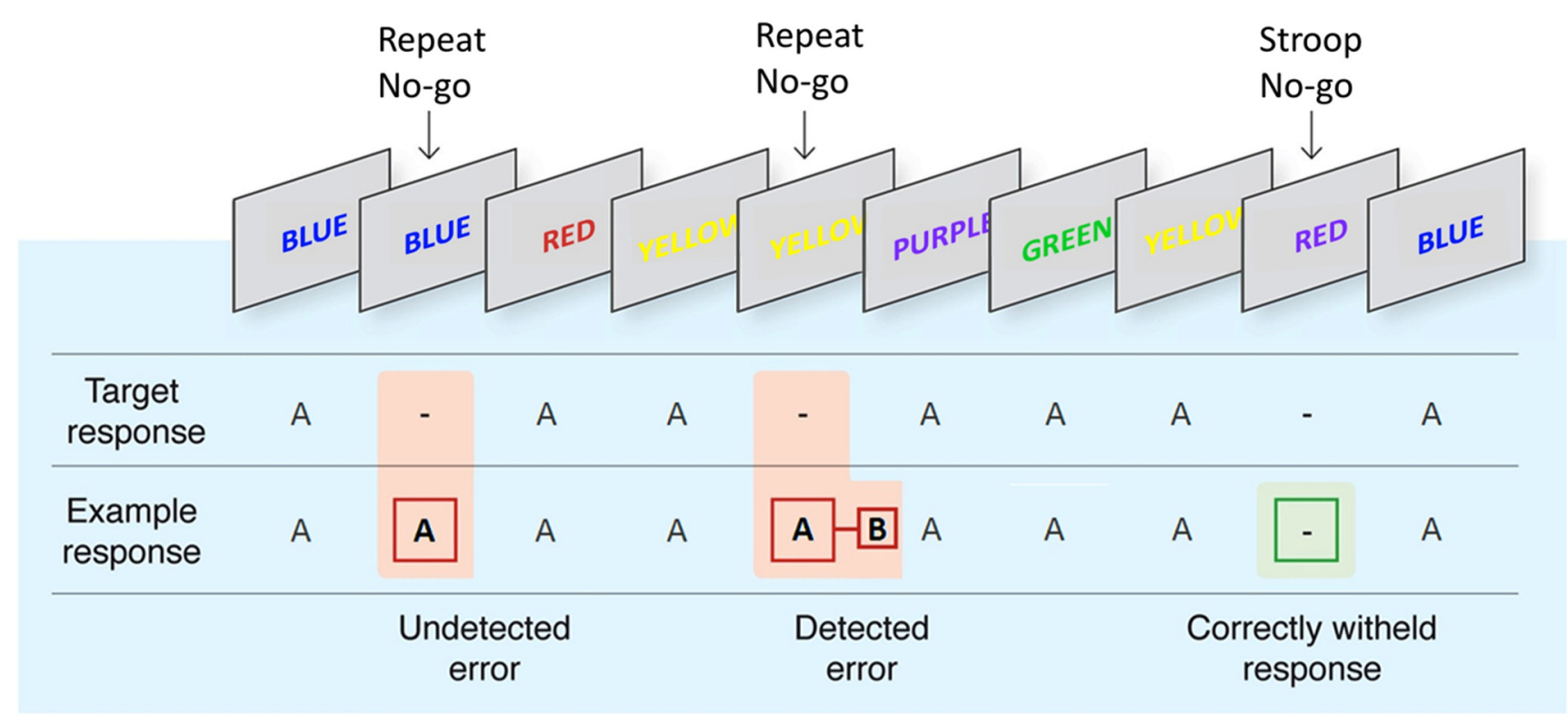

Figure 1. The EAT (Hester et al., 2005). Subjects were required to make a button press ("A") to all congruent stimuli (word and color matching) and to withhold from responding to incongruent stimuli (Stroop No-go) or when a word was repeated on consecutive trials (Repeat No-go). Subjects were also required to press a separate button ("B") as soon as possible after commission errors to signal error awareness.

Although there was a significant improvement in error awareness for Repeat No-go trials at the group level, we observed that there were a number of subjects for whom there was either no change, or a deterioration, with anodal tDCS (Fig. 3).

\section{Moderating factors}

Considering there was substantial variability in response to the intervention, we sought to examine whether factors, such as age or years of education, had a moderating role in the observed effects. Given the ceiling effects for Stroop No-go trials, response to the intervention was considered in terms of changes in error awareness for Repeat No-go trials only. There was no association between response to the intervention and age $(p=0.7)$, but there was a nonsignificant trend toward a positive correlation between response to the intervention and years of education $(r=0.278$, $p=0.055)$. We were also interested in examining whether the effects of the intervention varied as function of stimulation duration. The analysis of time on task effects revealed a main effect for Intervention $(p=0.008)$ and a main effect for half $(p=$ $0.001)$ such that error awareness for Repeat No-go trials decreased with time-on-task. There was no intervention $\times$ half interaction $(p=0.2)$.

\section{Sensations questionnaire}

The results of the sensations questionnaire indicated that all subjects tolerated the stimulation without discomfort. Although no strong sensations were reported, Real compared with Sham anodal stimulation was associated with greater levels of "itchiness" for the three anodal stimulation studies (all $p<0.05$ ). No other sensation was perceived to differ across the Real and Sham conditions (all $p>0.05$ ). To determine whether the perceived itchiness was associated with the performance-facilitating effect of tDCS, we examined the correlation between the intensity of the perceived itchiness and levels of error awareness at Real stimulation. This analysis revealed no significant association between perceived itchiness and overall error awareness or repeat error awareness for any of the experiments (all $p>0.05$ ). The results of the sensations questionnaire therefore indicate that perceived sensation did not influence performance.

\section{Discussion}

In the present study, we aimed to assess whether the application of tDCS over right DLPFC could modulate older adults' awareness of their performance errors. In two separate experiments, we demonstrated a reliable increase in the number of errors that were signaled by older adults during anodal stimulation to right DLPFC, relative to Sham stimulation. We observed increases of $12 \%$ and $10 \%$ in error awareness for Repeat No-go trials in Experiments 1 and 4, respectively. The improvement in error awareness could not be accounted for by changes in accuracy or slowed response times, as both of these task variables remained unchanged across Real and Sham stimulation conditions. We additionally demonstrated that, although anodal tDCS stimulation was effective when applied to right DLFPC, no change in performance was observed when left DLPFC was targeted. Our study therefore provides novel evidence that DLPFC regions subserving error awareness are predominantly lateralized to the right hemisphere.

The EAT involves two distinct No-go trial types: a design feature that is included to increase the overall error rate and hence to increase the probability that some errors will go unnoticed (Hester et al., 2005). tDCS-related improvements in error awareness were only evident for Repeat No-go trials. This observation merits consideration of whether tDCS affected a cognitive process that is specific to Repeat No-go trials. For instance, it may be argued that Repeat No-go relies more on memory than Stroop No-go, and the observed tDCS related improvements may therefore be the result of improved memory capacity. However, this does not reconcile with the fact that awareness and error rates are uncorrelated (O'Connell et al., 2009), that task difficulty and performance accuracy did not vary across Real and Sham conditions, and the observation that awareness on the EAT correlates with awareness of cognitive function in everyday life (Harty et al., 2013). A more plausible explanation for the absence of an effect 
A

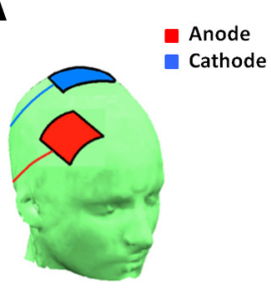

B

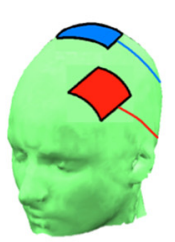

C

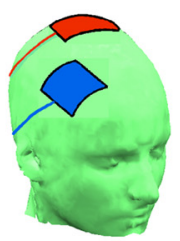

D

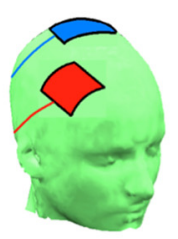

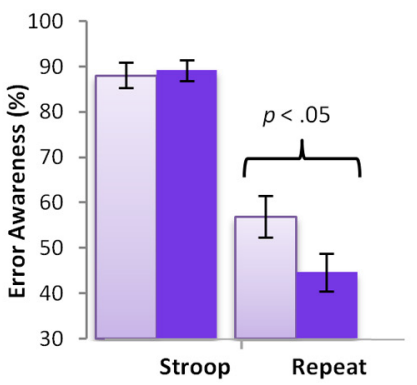
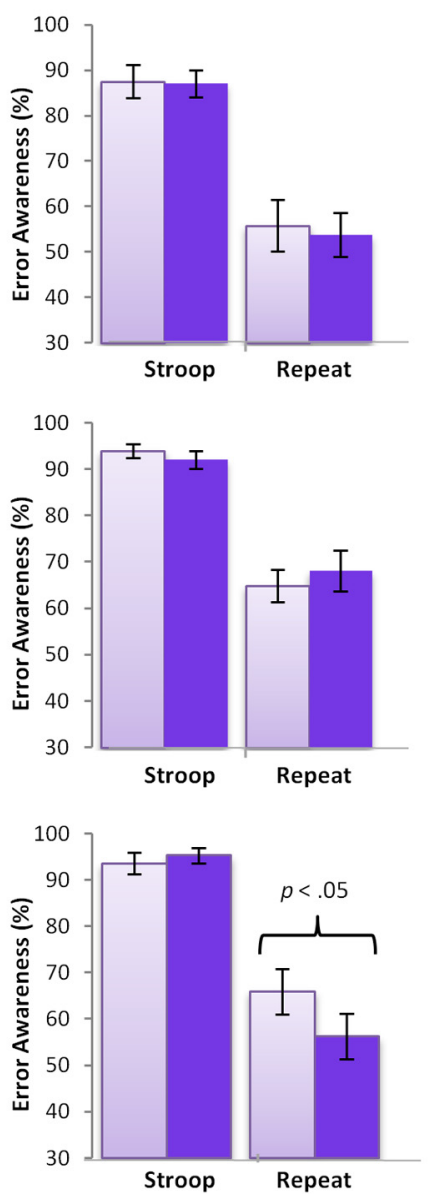

Figure 2. Effects of transcranial direct current stimulation (tDCS) on error awareness in Experiments 1-4. $\boldsymbol{A}$, Experiment 1: anodal tDCS over right dorsolateral prefrontal cortex (DLPFC) was associated with a significant improvement in error awareness for Repeat No-go trials. $\boldsymbol{B}$, Experiment 2: anodal tDCS over left DLPFC was not associated with any changes in error awareness. C, Experiment 3: cathodal tDCS over right DLPFC was not associated with any changes in error awareness. $D$, Experiment 4: the results of Experiment 1 were recapitulated in Experiment 4. Anodal tDCS over right DLPFC was again associated with a significant improvement in error awareness for Repeat No-go trials. Error bars represent SEM.

on Stroop No-go may be that performance was close to ceiling (average of 91\% across all experiments), likely leaving little room for any further improvements. Previous work suggests that tDCSinduced improvements may not be observed when baseline levels of performance are already high (Kang et al., 2009), and that facilitatory effects are more pronounced when there are greater levels of impairment (Hummel et al., 2006).

Cathodal stimulation over right DLPFC did not induce any behavioral changes, relative to Sham stimulation. This finding is consistent with several other studies that have failed to modulate cognitive functions using cathodal tDCS (Fregni et al., 2005; Cerruti and Schlaug, 2009; Fertonani et al., 2011; Friederici et al., 2013). A

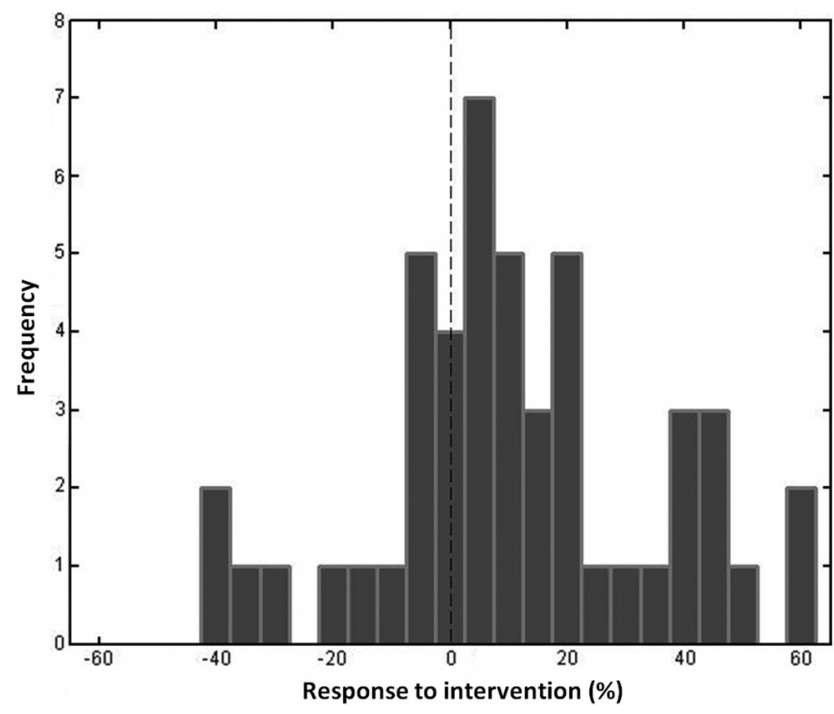

Figure 3. Histogram displaying individual variability in response to the intervention. Response to the intervention was defined as each subject's mean difference score between percentage error awareness for Repeat No-go trials at Real versus Sham stimulation conditions. Positive values indicate better error awareness for Repeat No-go trials at Real compared with Sham stimulation.

number of possible mechanisms have been proposed to explain the apparent resilience of cognitive functions to cathodal stimulation (for a review on theses aspects, see Miniussi et al., 2013). For instance, the significant cytoarchitectonic differences between neurons in the motor and frontal cortices are likely to play a role in the differential sensitivity of motor and cognitive functions to changes based on the direction of the current flow. The impact of tDCS may also depend on the initial state of neuronal activation. Although anodal stimulation might precipitate cognitive enhancement by boosting the excitability of neurons that by default are not reaching the threshold to contribute to the cognitive function, cathodal stimulation might not be sufficient to significantly impede the firing of neurons that are already activated by engaging in the cognitive task (Miniussi et al., 2013). The absence of cathodal stimulation effects on behavior might also reflect the compensatory recruitment of other regions within the cognitive networks (Jacobson et al., 2012).

Because tDCS is associated with sensations at the electrode site (Gandiga et al., 2006), there is a potential concern that greater somatic stimulation might induce expectancy effects. To investigate the relationship between expectancy and behavior, we examined the correspondence between perceived sensations induced by tDCS and error awareness at Real and Sham conditions. Although no strong sensations were reported, Real tDCS did induce more itchiness compared with Sham tDCS across Experiments 1, 2, and 4. However, there was no association between the intensity of subjects' perceived itchiness and levels of error awareness for Real tDCS, for any of the experiments. Furthermore, if perceived itchiness was the basis for the improvement in error awareness for Experiments 1 and 4, the perceived itchiness in Experiment 2 should have been associated with comparable improvements in error awareness. These findings indicate that the facilitatory effects of tDCS were not linked to perceived sensations or expectancy.

The finding that anodal tDCS produced an effect when applied over right, but not left, DLPFC provides new evidence for a dominant role of right DLPFC in mediating awareness. Although this finding is highly consistent with the clinical and brain lesion 
literature (Reed et al., 1993; Starkstein et al., 1995; Antoine et al., 2004; Shad et al., 2004, 2006; Harwood et al., 2005; Mendez and Shapira, 2005; Hoerold et al., 2013), it does not reconcile directly with event-related functional imaging work in which right DLPFC is often not implicated in error awareness (Debener et al., 2005; Hester et al., 2005, 2009; Klein et al., 2007). However, event-related functional imaging research to date has focused on error-evoked activations. Although this approach serves to isolate structures that are activated during the processing of an erroneous action, awareness may be largely determined by adaptive neural activity before target onset. For example, previous research has demonstrated a close relationship between the ability to maintain a vigilant state and error awareness (McAvinue et al., 2005, Shalgi et al., 2007; O'Keefe et al., 2007; Hoerold et al., 2013; Robertson, 2010; Harty et al., 2013). Nonetheless, it is clear that several other regions are implicated in error awareness, so it remains to be seen whether right DLPFC is the only stimulation site through which such gains can be achieved.

It is also important to consider the potential influence of changes in neural excitability under the reference electrode (e.g., Nitsche et al., 2007). Considering that no effects of stimulation were observed in Experiment 2, where the reference electrode was also placed over $\mathrm{Cz}$, the observed effects are unlikely to be the result of neuromodulatory changes in the vicinity of the reference electrode. In addition, the scalp location $\mathrm{Cz}$ corresponds to the confluence of the right and left central sulcus, which has not been implicated in any study of error awareness to date. That said, we cannot be sure about precisely how lateral or anterior the rightlateralized stimulation effects were. Although no modeling study to date has simulated the electrode montage used in the present study, studies have shown that similar montages result in diffuse current flow through the brain (Sadleir et al., 2010). Therefore, we cannot rule out the possibility that the performance facilitating effect of tDCS was mediated through stimulation of other brain regions aside from right DLPFC. It will be of value for future research to combine tDCS, neuroimaging, and modeling to help clarify how tDCS is influencing the underlying cortex and modulating error awareness.

It will also be important for future studies to examine the extent to which these findings generalize to populations other than older adults. Although there is compelling evidence of a strong association between the capacity for awareness and right DLPFC across several different populations, research also suggests that the natural aging process is associated with a general increase in frontal lobe recruitment (e.g., Davis et al., 2008). Considering this, it is important to acknowledge the possibility that the observed facilitatory effects of tDCS on error awareness may to some degree be attributable to functional reorganization in the aging brain.

Although the present study has demonstrated that a single session of anodal tDCS can result in immediate improvements in error awareness, the maintenance of the effect beyond the period of stimulation was not assessed. Further work will therefore be required to determine the viability of tDCS as a tool for the longterm remediation of awareness deficits. Encouragingly, previous studies have shown that, after five consecutive sessions of anodal tDCS, the induced gains can persist for at least several weeks after stimulation (Boggio et al., 2012). The translational potential of these findings is also presently limited by the substantial interindividual variability in response to the tDCS. Previous work has already highlighted several factors that may contribute to this kind of variability, including subject-specific anatomy (Datta et al., 2012), age (Ridding and Ziemann, 2010), and years of educa- tion (Berryhill and Jones, 2012). Improving our understanding of these, and other determinants, will be critical for the application of these findings to clinical rehabilitation.

Our findings have both theoretical and practical implications. From a theoretical perspective, our findings support the view that right lateralized DLPFC structures play a critical role in mediating awareness of cognitive performance. At the translational level, the observed facilitatory effect of tDCS may be fruitful as a preliminary step toward optimizing tDCS as a tool to enhance error awareness in older adults, as well as other clinical populations afflicted by error awareness deficits. Importantly, we have previously established that error awareness on the EAT is representative of awareness on real-word tasks (Harty et al., 2013). Prioritizing the remediation of error awareness deficits may indeed confer benefits to other cognitive functions by providing impetus to implement compensatory strategies, or fostering willingness to engage in, and adhere to, therapeutic interventions.

\section{References}

Antoine C, Antoine P, Guermonprez P, Frigard B (2004) Awareness of deficits and anosognosia in Alzheimer's disease. L'Encephale 30:570-577. CrossRef Medline

Berryhill ME, Jones KT (2012) tDCS selectively improves working memory in older adults with more education. Neurosci Lett 521:148-151. CrossRef Medline

Boggio PS, Ferrucci R, Mameli F, Martins D, Martins O, Vergari M, Tadini L, Scarpini E, Fregni F, Priori A (2012) Prolonged visual memory enhancement after direct current stimulation in Alzheimer's disease. Brain Stimul 5:223-230. CrossRef Medline

Cerruti C, Schlaug G (2009) Anodal transcranial direct current stimulation of the prefrontal cortex enhances complex verbal associative thought. J Cogn Neurosci 21:1980-1987. CrossRef Medline

Datta A, Truong D, Minhas P, Parra LC, Bikson M (2012) Inter-individual variation during transcranial direct current stimulation and normalization of dose using MRI-derived computational models. Front Psychiatry 3:91. CrossRef Medline

David AS, Bedford N, Wiffen B, Gilleen J (2012) Failures of metacognition and lack of insight in neuropsychiatric disorders. Philos Trans R Soc Lond B Biol Sci 367:1379-1390. CrossRef Medline

Davis SW, Dennis NA, Daselaar SM, Fleck MS, Cabeza R (2008) Qué PASA? The posterior-anterior shift in aging. Cereb Cortex 18:1201-1209. CrossRef Medline

Debener S, Ullsperger M, Siegel M, Fiehler K, von Cramon DY, Engel AK (2005) Trial-by-trial coupling of concurrent electroencephalogram and functional magnetic resonance imaging identifies the dynamics of performance monitoring. J Neurosci 25:11730-11737. CrossRef Medline

De Martino B, Fleming SM, Garrett N, Dolan RJ (2013) Confidence in value-based choice. Nat Neurosci 16:105-110. CrossRef Medline

Fertonani A, Pirulli C, Miniussi C (2011) Random noise stimulation improves neuroplasticity in perceptual learning. J Neurosci 31:1541615423. CrossRef Medline

Fleming SM, Weil RS, Nagy Z, Dolan RJ, Rees G (2010) Relating introspective accuracy to individual differences in brain structure. Science 329: 1541-1543. CrossRef Medline

Fleming SM, Huijgen J, Dolan RJ (2012) Prefrontal contributions to metacognition in perceptual decision making. J Neurosci 32:6117-6125. CrossRef Medline

Folstein M (1975) Mini-mental state a practical method for grading the cognitive state of patients for the clinician. London: Pergamon.

Fregni F, Boggio PS, Nitsche M, Bermpohl F, Antal A, Feredoes E, Marcolin MA, Rigonatti SP, Silva MT, Paulus W, Pascual-Leone A (2005) Anodal transcranial direct current stimulation of prefrontal cortex enhances working memory. Exp Brain Res 166:23-30. CrossRef Medline

Friederici AD, Mueller JL, Sehm B, Ragert P (2013) Language learning without control: the role of the PFC. J Cogn Neurosci 25:814-821. CrossRef Medline

Gandiga PC, Hummel FC, Cohen LG (2006) Transcranial DC stimulation (tDCS): a tool for double-blind sham-controlled clinical studies in brain stimulation. Clin Neurophysiol 117:845-850. CrossRef Medline

Harty S, O’Connell RG, Hester R, Robertson IH (2013) Older adults have 
diminished awareness of errors in the laboratory and daily life. Psychol Aging 28:1032-1041. CrossRef Medline

Harwood DG, Sultzer DL, Feil D, Monserratt L, Freedman E, Mandelkern MA (2005) Frontal lobe hypometabolism and impaired insight in Alzheimer disease. Am J Geriatric Psychiatry 13:934-941. CrossRef Medline

Hedden T, Gabrieli JD (2004) Insights into the ageing mind: a view from cognitive neuroscience. Nat Rev Neurosci 5:87-96. CrossRef Medline

Hester R, Foxe JJ, Molholm S, Shpaner M, Garavan H (2005) Neural mechanisms involved in error processing: a comparison of errors made with and without awareness. Neuroimage 27:602-608. CrossRef Medline

Hester R, Nestor L, Garavan H (2009) Impaired error awareness and anterior cingulate cortex hypoactivity in chronic cannabis users. Neuropsychopharmacology 34:2450-2458. CrossRef Medline

Hoerold D, Pender NP, Robertson IH (2013) Metacognitive and online error awareness deficits after prefrontal cortex lesions. Neuropsychologia 51:385-391. CrossRef Medline

Hummel FC, Voller B, Celnik P, Floel A, Giraux P, Gerloff C, Cohen LG (2006) Effects of brain polarization on reaction times and pinch force in chronic stroke. BMC Neurosci 7:73. CrossRef Medline

Jacobson L, Koslowsky M, Lavidor M (2012) tDCS polarity effects in motor and cognitive domains: a meta-analytical review. Exp Brain Res 216:1-10. CrossRef Medline

Kang EK, Baek MJ, Kim S, Paik NJ (2009) Non-invasive cortical stimulation improves post-stroke attention decline. Restor Neurol Neurosci 27:645650. CrossRef Medline

Klein TA, Endrass T, Kathmann N, Neumann J, von Cramon DY, Ullsperger M (2007) Neural correlates of error awareness. Neuroimage 34:17741781. CrossRef Medline

McAvinue L, O'Keeffe F, McMackin D, Robertson IH (2005) Impaired sustained attention and error awareness in traumatic brain injury: implications for insight. Neuropsychol Rehabil 15:569-587. CrossRef Medline

Mendez MF, Shapira JS (2005) Loss of insight and functional neuroimaging in frontotemporal dementia. J Neuropsychiatry Clin Neurosci 17:413416. CrossRef Medline

Miniussi C, Harris JA, Ruzzoli M (2013) Modelling non-invasive brain stimulation in cognitive neuroscience. Neurosci Biobehav Rev 37:17021712. CrossRef Medline

Murphy PR, Robertson IH, Allen D, Hester R, O'Connell RG (2012) An electrophysiological signal that precisely tracks the emergence of error awareness. Front Hum Neurosci 6:65. CrossRef Medline

Nitsche MA, Doemkes S, Karaköse T, Antal A, Liebetanz D, Lang N, Tergau F, Paulus W (2007) Shaping the effects of transcranial direct current stimulation of the human motor cortex. J Neurophysiol 97:3109-3117. CrossRef Medline

Nitsche MA, Cohen LG, Wassermann EM, Priori A, Lang N, Antal A, Paulus W, Hummel F, Boggio PS, Fregni F, Pascual-Leone A (2008) Transcranial direct current stimulation: state of the art 2008. Brain Stimul 1:206223. CrossRef Medline

O'Connell RG, Dockree PM, Bellgrove MA, Kelly SP, Hester R, Garavan H, Robertson IH, Foxe JJ (2007) The role of cingulate cortex in the detec- tion of errors with and without awareness: a high-density electrical mapping study. Eur J Neurosci 25:2571-2579. CrossRef Medline

O'Connell RG, Bellgrove MA, Dockree PM, Lau A, Hester R, Garavan H, Fitzgerald M, Foxe JJ, Robertson IH (2009) The neural correlates of deficient error awareness in attention-deficit hyperactivity disorder (ADHD). Neuropsychologia 47:1149-1159. CrossRef Medline

O'Keeffe FM, Dockree PM, Moloney P, Carton S, Robertson IH (2007) Characterising error-awareness of attentional lapses and inhibitory control failures in patients with traumatic brain injury. Exp Brain Res 180: 59-67. CrossRef Medline

Poreisz C, Boros K, Antal A, Paulus W (2007) Safety aspects of transcranial direct current stimulation concerning healthy subjects and patients. Brain Res Bull 72:208-214. CrossRef Medline

Rabbitt P (1990) Age, IQ and awareness, and recall of errors. Ergonomics 33:1291-1305. CrossRef Medline

Rabbitt P (2002) Consciousness is slower than you think. Q J Exp Psychol A 55:1081-1092. CrossRef Medline

Raz N (2004) The aging brain: structural changes and their implications for cognitive aging. Oxford: Oxford UP

Reed BR, Jagust WJ, Coulter L (1993) Anosognosia in Alzheimer's disease: relationships to depression, cognitive function, and cerebral perfusion. J Clin Exp Neuropsychol 15:231-244. CrossRef Medline

Ridding MC, Ziemann U (2010) Determinants of the induction of cortical plasticity by non-invasive brain stimulation in healthy subjects. J Physiol 588:2291-2304. CrossRef Medline

Robertson IH (2010) Anosognosia and error processing in various clinical disorders. In: Study of anosognosia (Prigatano GP, ed.), pp 321-331. Oxford: Oxford UP.

Rossi S, Hallett M, Rossini PM, Pascual-Leone A (2009) Safety, ethical considerations, and application guidelines for the use of transcranial magnetic stimulation in clinical practice and research. Clin Neurophysiol 120:2008-2039. CrossRef Medline

Ruff CC, Ugazio G, Fehr E (2013) Changing social norm compliance with noninvasive brain stimulation. Science 342:482-484. CrossRef Medline

Sadleir RJ, Vannorsdall TD, Schretlen DJ, Gordon B (2010) Transcranial direct current stimulation (tDCS) in a realistic head model. Neuroimage 51:1310-1318. CrossRef Medline

Schulz R, Gerloff C, Hummel FC (2013) Non-invasive brain stimulation in neurological diseases. Neuropharmacology 64:579-587. CrossRef Medline

Shad MU, Muddasani S, Prasad K, Sweeney JA, Keshavan MS (2004) Insight and prefrontal cortex in first-episode schizophrenia. Neuroimage 22: 1315-1320. CrossRef Medline

Shad MU, Muddasani S, Keshavan MS (2006) Prefrontal subregions and dimensions of insight in first-episode schizophrenia: a pilot study. Psychiatry Res 146:35-42. CrossRef Medline

Shalgi S, O'Connell RG, Deouell LY, Robertson IH (2007) Absent minded but accurate: delaying responses increases accuracy but decreases error awareness. Exp Brain Res 182:119-124. CrossRef Medline

Starkstein SE, Vázquez S, Migliorelli R, Tesón A, Sabe L, Leiguarda R (1995) A single-photon emission computed tomographic study of anosognosia in Alzheimers disease. Arch Neurol 52:415-420. CrossRef Medline 